\title{
Archéopages
}

Archéopages

Archéologie et société

$46 \mid 2018$

Maisons

\section{Dépôt de fondation conventuelle}

\section{Mathieu Ott et Jean-Louis Vayssettes}

\section{OpenEdition}

Journals

Édition électronique

URL : https://journals.openedition.org/archeopages/4603

DOI : 10.4000/archeopages.4603

ISSN : 2269-9872

\section{Éditeur}

INRAP - Institut national de recherches archéologiques préventives

\section{Édition imprimée}

Date de publication : 1 décembre 2018

Pagination : 106-107

ISSN : 1622-8545

\section{Référence électronique}

Mathieu Ott et Jean-Louis Vayssettes, « Dépôt de fondation conventuelle », Archéopages [En ligne],

46 | 2018, mis en ligne le 01 décembre 2020, consulté le 02 juin 2021. URL : http://

journals.openedition.org/archeopages/4603; DOI : https://doi.org/10.4000/archeopages.4603 


\section{Dépôt de fondation conventuelle}

\author{
Mathieu Ott \\ Inrap, UMR 5140 " Archéologie des Sociétés \\ Méditerranéennes", responsable d'opération \\ Jean-Louis Vayssettes \\ Ministère de la Culture
}

Site
Couvent des Récollets
Montpellier
Hérault
Date
Septembre 2016
Surface
$3086 \mathrm{~m}^{2}$
Équipe
Infographie
Frédérique Robin
Inrap

en 1663. La construction est menée entre 1664 et 1666 , et, en 1689 ,

la chapelle est bénie. La façade, de facture classique, est achevée en 1738. En 1790, l'ordre des Récollets est supprimé, leurs biens sont saisis et vendus. Au cours du XIX ${ }^{\mathrm{e}}$ siècle, les bâtiments abritent successivement l'annexe de la prison de Montpellier, puis le Grand Séminaire, enfin les Archives départementales de l'Hérault jusqu'à leur destruction en 1977. Seule la chapelle a été préservée [ill. 1].

Le diagnostic archéologique a confirmé l'éradication des vestiges bâtis, rasés jusqu'au niveau des fondations. L'expertise a en revanche révélé l'omniprésence des tombes, que ce soit dans le cloître ou à l'intérieur des bâtiments. Le couvent a servi de lieu d'inhumation non seulement aux frères Récollets, mais aussi à une partie de la population de Montpellier, entre la fin du XVII ${ }^{\mathrm{e}}$ siècle et le milieu du $\mathrm{XIX}^{\mathrm{e}}$ siècle. En témoignent les textes, que corroborent les premières observations archéothanatologiques.

Surtout, le diagnostic a permis la mise au jour d'une fosse contenant in situ un coffre monolithe d'environ $300 \mathrm{~kg}$, au couvercle scellé au mortier [ill. 2]. À l’intérieur, enchâssée dans la pierre [ill. 3], une plaque de plomb carrée de 36,7 cm de côté présentait, gravé sur son revers, un texte relatant la pose de la première pierre du couvent des frères mineurs Récollets par l'évêque de Montpellier [ill. 4] :

Au nom de Notre Seigneur Jésus Christ

Ici est posée la première pierre de l'église des frères mineurs Récollets, sous la titulature de la Sainte Famille Jésus Marie Joseph, par l'illustrissime et révérendissime seigneur évêque de Montpellier François de Bosquet, son fondateur, et par les seigneurs Jean de Sartre, conseiller du roi en la cour des comptes et aides de Languedoc et Brigitte de Massane, sa femme, par Jacques de Grille, gouverneur royal de la cité d'Arles et Delphine de Sartre, son épouse, sous le pontificat du pape Alexandre VII, régnant Louis XIV, Gaspard de Lacroix, marquis de Castries, chevalier à torque, étant gouverneur de la citadelle et de la ville, étant consuls les très nobles et illustres Charles de Pélissier de Boirargues, François Coste, François Satgier, Antoine Amouroux, le jour $5^{e}$ d'octobre de l'année 1664.

La cérémonie de la pose de la première pierre du couvent a en outre été relatée par un témoin del'événement. Ce texte, publié en 1896 (Flament, 1896, p. 21-22), conforte et illustre les constatations archéologiques : François de Bosquet « fit procéder au solennel posement de la pierre fondamentale de ce couvent, [...] à la veue de plus de dix mille personnes, tant catholiques qu'huguenots [...] Messieurs les Consuls en robbe rouge, [...] y parurent à costé dudict Seigneur Evesque, suivy de presque tout son clergé, qui posa de ses propres mains la première pierre de ce futur sanctuaire [...]. Les acclamations feurent si générales de tous les endroicts qu'il ne s'estoit pas veu despuis longtemps une si solennelle action dans Montpellier, ny moins si généralement applaudie de tout le monde aussy bien que de tout le voisinage, qui en feut adverty par le bruit des canons de la citadelle [...]». Montpellier connaît en effet depuis 1622 une éphémère période de tranquillité, qui dure jusqu'à la révocation de l'édit de Nantes en 1685. Le texte souligne non sans une certaine emphase l'importance de la cérémonie. Elle rassemblait tous les corps religieux civils et militaires qui symbolisent la mainmise de l'absolutisme monarchique et catholique sur une ville protestante.

La plaque s'ajoute à une série très restreinte de dépôts enfouis au moment de la fondation de plusieurs édifices montpelliérains : l'église de l'Observance en 1605 , la chapelle des Carmes du Palais en 1706, l'hôtel de Saint-Félix en 1751, le château de la Mosson, Notre-Dame-des-Tables... Autant de précieux documents sur l'histoire de Montpellier. La plaque des Récollets reste cependant la seule mise au jour dans son contexte archéologique. Elle montre la parfaite concordance entre les textes et les archives du sol. 
d'inhumations dans cette

partie des bâtiments

conventuels.

3. Gravés dans le bloc

calcaire, les symboles

chrétiens mis au jour lors

de la dépose de la plaque

de plomb.

4. La plaque mesure $3 \mathrm{~mm}$

d'épaisseur, pour un poids

de 4,135 kg.Anépigraphe sur

son avers, elle présente au

revers un texte gravé sur

13 lignes. La régularité de

l'inscription a été assurée

par un travail préparatoire :

23 incisions horizontales

parfaitement rectilignes

et parallèles, distantes de

1,4 à $1,6 \mathrm{~cm}$, ont été finement

réalisées à la règle et à la

pointe sèche. Les caractères

sont également gravés à la

pointe sèche mais à main

levée-lls mesurent 1,2 à

$1,6 \mathrm{~cm}$ de haut et sont très réguliers.

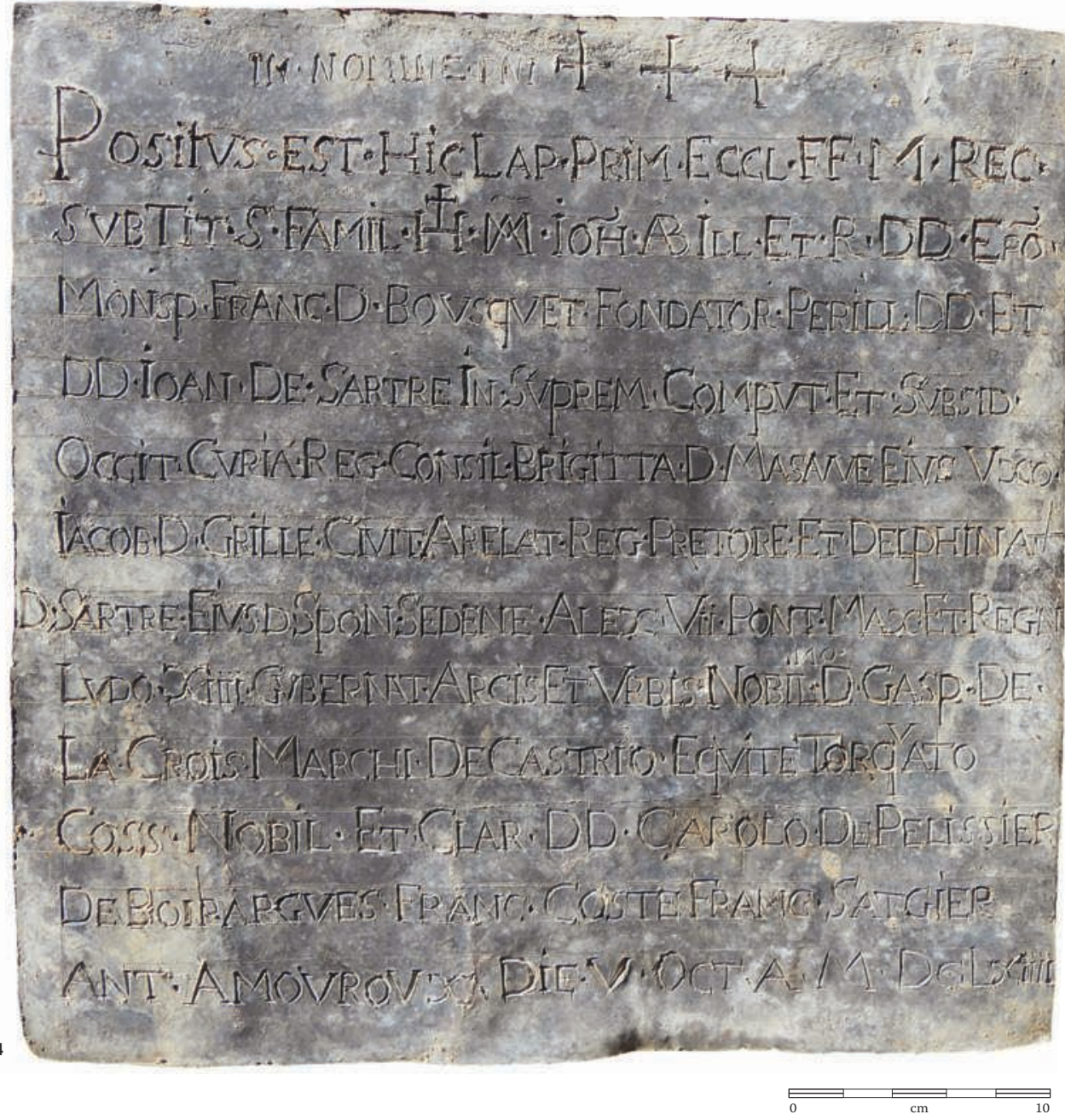

ఏ
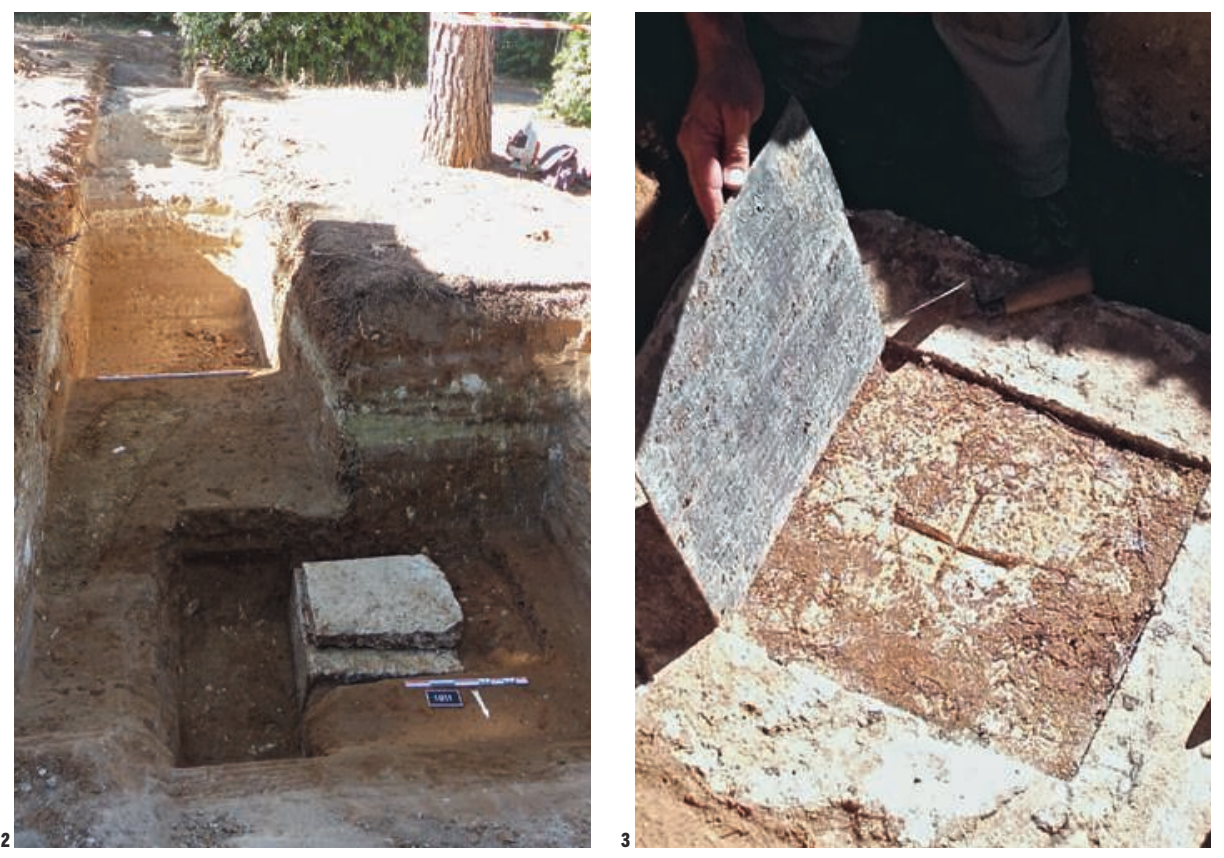

Références bibliographiques

AigrefEUILLE C. d' 1739, Histoire de la ville de Montpellier, seconde partie contenant l'origine de son église, la suite de ses évêques, ses églises particulières, ses monastères anciens $\mathcal{E}$ modernes, ses hôpitaux...

Montpellier, Rigaud père \& fils, $455 \mathrm{p}$. Flament M., 1896, Notice sur la chapelle et les bâtiments du Grand Séminaire de Montpellier, Montpellier, impr. Firmin et Montané, $125 \mathrm{p}$. 